Revue d'histoire de l'enfance « irrégulière »

Le Temps de l'histoire

4 | 2002

Images de l'enfance et de la jeunesse "irrégulières »

\title{
Archéologie de l'Apachisme. Les représentations des Peaux-Rouges dans la France du XIXe siècle
}

Dominique Kalifa

\section{(2) OpenEdition \\ Journals}

Édition électronique

URL : http://journals.openedition.org/rhei/51

DOI : $10.4000 /$ rhei.51

ISBN : 978-2-7535-1642-7

ISSN : $1777-540 \mathrm{X}$

Éditeur

Presses universitaires de Rennes

Édition imprimée

Date de publication : 15 novembre 2002

Pagination : 19-37

ISSN : 1287-2431

Référence électronique

Dominique Kalifa, «Archéologie de l'Apachisme. Les représentations des Peaux-Rouges dans la

France du XIXe siècle », Revue d'histoire de l'enfance « irrégulière » [En ligne], 4 | 2002, mis en ligne le 16 juillet 2007, consulté le 04 décembre 2020. URL : http://journals.openedition.org/rhei/51 ; DOI : https://doi.org/10.4000/rhei.51 


\section{Archéologie de l'Apachisme. \\ Les représentations des Peaux-Rouges dans la France du xIXème siècle}

On sait la fortune du terme Apache, lancé dès l'été 1900 pour désigner les jeunes voyous de la capitale, et bientôt étendu à tous les délinquants juvéniles, voire à tous les criminels du pays. ${ }^{(2)}$ Suscitant presque d'emblée une véritable débauche discursive, ce mot et ses innombrables dérivés (apachisme, apacherie, apachocratie, etc.) firent de la Belle Époque un étonnant «moment apache " de l'Histoire de France. "On ne parle que d'apaches", ${ }^{(3)}$ titre sans ironie un journaliste de 1910. Passées les grandes émotions du début du siècle, le terme résista assez bien dans la presse et la culture de grande diffusion, au moins durant l'entre-deuxguerres. ${ }^{(4)}$ Il perdura plus longtemps dans l'imaginaire pittoresque, périodiquement réactivé par le cinéma, la chanson, la littérature anecdotique ou le roman policier. Une énigme pourtant pesait sur sa naissance. L'acte de baptême n'était pas signé, et c'est presque rituellement que l'on s'interrogeait à chaque résurgence sur les origines précises du terme. La filiation lointaine ne posait guère de problème : on citait Fenimore Cooper, Gustave Aimard et l'engouement du tournant du siècle pour l'Ouest américain. L'ascendance immédiate suscitait en revanche les discussions. Certains attribuaient la paternité du terme à "un spirituel chroniqueur du Palais ", ${ }^{(5)}$ d'autres à des journalistes (Victor Morris, chef des Informations au Matin, ou Arthur Dupin, son alter ego au Journal ${ }^{(6)}$ ), d'autres encore y voyaient la main du secrétaire du commissariat de Belleville ou estimaient que le terme avait jailli spontanément dans le milieu des rôdeurs de l'Est parisien.(7) Controverse subtile, mais interminable, dans la mesure où chacune des propositions risque fort de se révéler exacte et la création collective. On voudrait donc ici inverser les perspectives, en soulignant à l'inverse combien les vrais enjeux du terme

\section{Dominique} Kalifa
(1) Professeur à

l'université Rennes-2/ CRHISCO.

(2) C'est à Michelle Perrot que l'on doit la découverte historiographique des Apaches. Elle dirigea le premier travail universitaire qui leur soit consacré (Laurent Cousin, Les Apaches. Délinquance juvénile à Paris au début du XXème siècle, maîtrise d'histoire, université de Paris-7, 1976) et rédigea peu après "Dans le Paris de la Belle Époque, les Apaches, premières bandes de jeunes" (Les marginaux et les exclus dans l'Histoire, 1979), repris dans Les ombres de l'Histoire. Crime et chatiment au XIXème siècle, 
Paris, Flammarion, 2001, p. 351-364. J'ai prolongé, pour ma part, certains points dans L'encre et le sang. Récits de crimes et société à la Belle Époque, Paris, Fayard, 1995.

(3) Le Matin, 12 janvier 1910.

(4) Vanessa Zerjav, La pègre parisienne dans les années vingt, maîtrise d'histoire, université de Paris-7, 1998.

(5) Paul Matter, "Chez les Apaches", Revue politique et littéraire, octobre 1907, p. 626.

(6) Louis Latzarus,

"Les malfaiteurs parisiens", Revue de Paris, 1er juin 1912, p. 527 ;

Marcel Montarron,

Histoire du milieu de Casque d'or à nos jours, Paris, Plon, 1969 , p. 19.
(7) Ernest Laut, Supplément illustré du Petit journal, 23 juin 1910 ; Le Matin, 31 août 1900.

(8) George D. Morris, Fenimore Cooper et Edgar Poe d'après la critique française du XIXème siècle, Paris, Larose,

1912 ; Margaret Murray Gibb, Le roman de Basde-cuir. Étude sur Fenimore Cooper et son influence en France, Paris, Champion, 1927 ; Georgette Bosset, Fenimore Cooper et le roman d'aventures en France vers 1830, Paris, Vrin, 1928.

(9) Les pionniers (1825), Le dernier des Mohicans (1826), La prairie (1827), Le lac Ontario (1840),

Le tueur de daims (1841).

L'ensemble, paru d'abord chez Baudry, Bossange, Galignani, fut repris par Gosselin, qui entama en 1827 la publication des Euvres complètes de James Fenimore Cooper. résident plutôt dans le recours à l'exotisme indien et aux transferts qu'il mobilise. Né à la croisée des "Deux-Mondes", produit du "populaire" tout autant que du "savant", l'Apache interroge le XIXème siècle sur quelques-unes de ses contradictions (civilisation versus sauvagerie, optimisme vs anxiété, espace urbain $v$ s espaces vierges), tout en soulignant la part croissante prise par l'imaginaire américain dans la formulation et la résolution des nouveaux dysfonctionnements sociaux.

\section{Le sauvage et le prolétaire}

C'est entre la fin de la Restauration et les premières années de la Monarchie de Juillet que se met en place la configuration propre à l'Apache. Sans doute le terme est-il attesté depuis longtemps. L'Encyclopédie évoque dès 1751 ces "peuples de l’Amérique septentrionale au Nouveau Mexique », et plusieurs voyageurs citent leur nom au hasard de leurs pérégrinations. Mais ce qui survient entre 1827 et 1831 n'a que peu de rapports avec la lexicologie ou les attestations linguistiques ; au vrai, le mot Apache n'y fut peut-être même pas prononcé. Une convergence pourtant s'y dessine, qui rend possibles les futurs usages du terme. Deux événements y concourent, qui n'entretiennent entre eux aucune relation apparente : d'une part, l'immense engouement littéraire que suscitent alors les romans et l'imaginaire de Fenimore Cooper, de l'autre, la grande frayeur sociale engendrée par la misère prolétaire et l'insurrection des canuts lyonnais en novembre 1831. Sur l'influence de Cooper à la fin de la Restauration, tout ou presque a été dit par les trois thèses consacrées au sujet au début du Xxème siècle. ${ }^{(8)}$ On sait que l'Amé- 
ricain, qui réside en France de 1826 à 1833, suscite alors littéralement l'événement. Aussitôt traduits, les romans du cycle de Bas-de-cuir ${ }^{(9)}$ reçoivent un accueil enthousiaste de la critique, et tout ce que le pays compte alors de "classe pensante" se passionne pour " le Walter Scott des sauvages ". ${ }^{(10)}$ Sainte-Beuve, Dumas, George Sand, Maxime du Camp, Eugène Sue, Béranger et beaucoup vibrent alors avec Le dernier des Mohicans, tandis que Balzac, le plus fervent des admirateurs de Cooper, transpose son univers dans de multiples romans, à commencer par Les chouans. Autour des Mohawks, des Iroquois, des Onondagas, des Delaware et des nombreuses autres "races rouges " dépeintes par Cooper, s'organise un premier "moment indien ». En 1827, des Osages en visite à Paris font l'objet d'une série d'observations. ${ }^{(1)}$ Quelques années plus tard, en 1833, ce sont des Indiens charruas qui sont présentés à l'Académie des sciences.

C'est dans ce contexte que surviennent les événements lyonnais de 1831, qui donnent leur pleine mesure à cette hantise du "barbare" qui taraudait depuis une vingtaine d'années un imaginaire social nourri de romantisme et de saint-simonisme. ${ }^{(12)}$ Incarnation de tous les périls, cette antique figure du désordre resurgit alors sous l'effet des nouvelles problématiques sociales. Pensés comme une population fondamentalement hostile et inassimilable, prolétaires et plébéiens sont alors représentés comme des hordes sauvages et déferlantes. Alors que les historiens relisent l'épisode des grandes invasions et la naissance du MoyenÂge, journalistes et publicistes diffusent l'image d'une civilisation en crise, bourrelée de frayeurs obsidionales, menacée par l'assaut des nouveaux barbares, prolétaires, sauvages, criminels et exclus de toute sorte. Mais des barbares plus redoutables que leurs aînés, parce qu'ennemis de l'intérieur d'abord, parce que produits surtout d'une civilisation en marche et à l'avènement de laquelle ils sont indispensables. L'immense retentissement que connaît l'article de Saint-Marc Girardin dans Le Journal des débats du 8 décembre 1831 exprime assez bien la prégnance de cet imaginaire et de ses apories.

$\mathrm{Si}$ les Indiens dont on se soucie sont alors des Iroquois ou des Hurons, et non des Apaches, on perçoit cependant toute l'importance que revêt cette séquence dans la genèse du phénomène. Une inflexion

(10) Le Globe, 24 mai 1827. Sur ce milieu, voir Jean-Jacques Goblot, La jeune France libérale. Le Globe et son groupe littéraire (1824-1830), Paris, Plon, 1995.

(11) Paul Valcourt, Histoire de la tribu des Osages, peuplade sauvage de l'Amérique septentrionale, dans l'État du Missouri, écrite par six Osages actuellement à Paris, par M. P. Valcourt, suivie de la relation $d u$ voyage de ces sauvages et d'une notice historique sur chacun de ces Indiens, Paris, Béchet, 1827.

(12) Pierre Michel, Un mythe romantique : les Barbares, 1789-1848, Presses universitaires de Lyon, 1981. Voir aussi Louis Chevalier, Classes laborieuses et classes dangereuses à Paris pendant la première partie du XIXème, Paris, Plon, 1958, p. 593-613 ; et JeanClaude Beaune et al., 
Les sauvages dans la Cité. Auto-émancipation du peuple et instruction des prolétaires au XIXème siècle, Seyssel, Champ Vallon, 1985.

(13) Barret Wendell, $A$ Literary History of America, Londres, 1891, p. 185, cité par M. Gibb, Le roman de Bas-de-cuir, op. cit., p. 78-79.

(14) Voir, sur ce point, les travaux de G.

Chinard, L'exotisme américain dans la littérature

française au XVIème siècle d'après Rabelais, Ronsard, Montaigne, etc., Paris, Hachette, 1911 ; L'Amérique et le rêve exotique dans la littérature française du XVIIème siècle, Paris, 1913 ; L'exotisme américain dans l'œuvre de Chateaubriand, Paris, 1918. Pour une perspective d'ensemble et plus récente : R. J. Berkhofer Jr, The White Man's Indian. Images of the American Indian from
Colombus to the Present, New York, Alfred, Knopf, 1978.

(15) H. Liebersohn, Aristocratic Encounters. European Travellers and North American Indians, London, Cambridge University Press, 1998.

(16) RP JosephFrançois Lafitau, Moeurs des sauvages américains, comparées aux mours des premiers temps (1724),

Paris, Maspéro, 1983.

(17) Jean-René-

Constant Quoy et Paul Gaimard, Voyage autour $d u$ monde, 1824, cité par Marc Renneville, Le langage des crânes. Une bistoire de la phrénologie, Paris, Synthélabo, 2000, p. 54.

\section{(18) Lucienne}

Frappier-Mazur, L'expression métaphorique dans la Comédie bumaine, Paris, Klincksieck, 1974, p. 150. importante est en effet donnée à la représentation de l'Indien d'Amérique, qui cesse alors peu à peu d'être un "bon sauvage". La remarque ne vaut pas pour Cooper, dont " les nobles Indiens ressemblent en effet plutôt aux rêves de la France du XVIIIème siècle sur la nature humaine primitive qu'à ce qu'ont pu chercher les ethnographes critiques ", ${ }^{(13)}$ même si le cycle fourmille aussi d'Indiens féroces comme Magua et de "maudits Iroquois ". Mais l'émergence conjointe des tribus d'Amérique et des barbares des faubourgs, l'association qui en résulte entre les sauvages de l'extérieur et ceux de l'intérieur, entre la frontière et les faubourgs, contribue au déclin progressif de l'image du bon sauvage. Invention de la France humaniste, cette figure restait pourtant assez vive chez les lettrés, où elle s'alimentait à de multiples traditions, ${ }^{(14)}$ celle de l'exotisme américain, support privilégié depuis le XVIIIème siècle des digressions philosophiques ou des critiques de la civilisation, celle du voyage et de l'imaginaire romantique qui perdure largement (Les Natchez de Chateaubriand paraît en 1826), celle des méditations sur la " noblesse naturelle " de certains peuples, qui nourrit souvent les récits de voyage des aristocrates du premier XIXème siècle. ${ }^{(15)}$ En 1845, on réédite encore les Mours des sauvages américains de Lafitau, qui contribua pour beaucoup à la diffusion, au XVIIIème siècle, des représentations du bon sauvage d'Amérique, Algonquins ou Iroquois. ${ }^{(16)}$ Mais ces images perdent de leur puissance face à la figure beaucoup plus inquiétante du prolétaire comme sauvage et aux critiques émanant des naturalistes et des anthropologues, qui fustigent « les paradoxes de ces philosophes chagrins qui, s'indignant des vices 
de l'homme en société, ont inventé l'homme de la nature tel qu'il n'existe pas ».(17) La littérature suit, qui se débarrasse peu à peu de ces images vieillies. «Le bon sauvage est une espèce qui n’a pas cours dans la Comédie humaine », ${ }^{(18)}$ affirme la critique balzacienne.

En revanche, progressent les représentations assimilant les barbares de l'intérieur aux Indiens d'Amérique. Déjà Châteaubriand, dans Le voyage en Amérique (1827), avait suggéré que la détresse des tribus indiennes égalait celle des prolétaires et de nombreux enquêteurs, comme Eugène Buret, développent l'idée selon laquelle "l'extrême misère est une rechute en sauvagerie $»{ }^{(19)}$ Mais Balzac est vraiment le premier à filer la métaphore, non dans le sens du paupérisme, mais dans celui de la violence et de la dangerosité. Ainsi évoque-t-il, dans son Code des gens honnêtes (1825), ces « sauvages " qui cernent Paris, avant d'exposer clairement la situation dans Le Père Goriot (1835) : "Paris, voyez-vous, est comme une forêt du Nouveau Monde, où s'agitent vingt espèces de peuplades différentes, les Illinois, les Hurons, qui vivent du produit que donnent les différentes chasses sociales. ${ }^{(20)}$ En décrivant la démocratie américaine entourée et menacée par les sauvages, l'œuvre de Tocqueville renforce cet usage, qui devient un véritable lieu commun au début des années 1840. La civilisation est un front pionnier, qui progresse entourée de tribus sauvages et hostiles. Massés dans l'ombre aux marges des cités, les rôdeurs de barrières sont-ils autre chose que des « cannibales de notre civilisation » ? ${ }^{(21)}$

L'immense succès public des Mystères de Paris, que publie Eugène Sue en 1842-1843, ancre ces figures dans un imaginaire social qui déborde désormais l'univers des seuls lettrés. Disciple de Cooper autant que de Saint-Marc Girardin, Sue abat en effet les dernières cloisons entre le sauvage d'Amérique, le prolétaire et le délinquant. « Nous allons essayer de mettre sous les yeux du lecteur quelques épisodes de la vie d'autres barbares aussi en dehors de la civilisation que les sauvages peuplades si bien peintes par Cooper. " ${ }^{(2)}$ Mais il donne à cette représentation une épaisseur inédite. D’abord parce que les tribus en question, au contraire des Iroquois, ne se contentent pas de rôder aux frontières : elles « sont au milieu de nous; nous pouvons les coudoyer en nous aventurant
(19) Cité par P.

Michel, Un mythe roman-

tique, op. cit., p. 213.

L'enquête d'Eugène

Buret (La misère des

classes laborieuses en France et en Angleterre), plus tardive, date de 1840

(20) Honoré de Balzac, Le Père Goriot, Paris, Gallimard, 1979, p. 279.

(21) Roland Bauchery, Les bohémiens de Paris, 1845, p. 40, cité par Simone Delattre, Les douze heures noires. La nuit à Paris au XIXème siècle, Paris, Albin Michel, 2000, p. 495.

(22) Eugène Sue, Les mystères de Paris (1842-1843), Paris, Pauvert, 1963, p. 7. 
(23) Henri Cauvain, Maximilien Heller (1871), Paris, Garnier, 1978, p. 96.

(24) George Sand, Relation d'un voyage chez les sauvages de Paris (1846), Paris, Michel Lévy, 1857, p. 282.

(25) Alexandre

Renaud, Au Congrès et au peuple des États-Unis.

Pour la protection des tribus indiennes d'Amérique $d u$ Nord, Paris,

Duverger, 1847.

(26) George Catlin,

La vie chez les Indiens.

Scènes et aventures de voyage parmi les tribus des deux Amériques, Paris, Hachette, 1863.

L'ouvrage évoque cependant les Apaches au passage (p. 46).

(27) Alexandre Dumas, Les Mobicans de Paris, (1854-1859), Paris, Gallimard, 1998.
(28) Ce qui différencie également les Français des autres observateurs européens, comme l'Irlandais

Thomas Mayne-Raid ou l'Allemand Friedrich Gerstacker. Voir sur ce point Tangi Villerbu,

"L’Amérique, une expérience européenne : aventuriers-romanciers du XIXème siècle”, communication au 125ème Congrès du CTHS, Lille, avril 2000 (à paraître).

(29) Voir Nancy N. Barker, "Voyageurs français au Mexique, fourriers de l'intervention (1830-1860)", Revue d'histoire diplomatique, 1973, p. 96-114. Sur la politique impériale au Mexique, voir Christian Schefer, $L a$ grande pensée de Napoléon III. Les origines de l'expédition au Mexique (1858-1862), Paris, Marcel Rivière, 1939 ; et Jean Avenel, La cam- dans les repaires où ils vivent, où ils se rassemblent pour concerter le meurtre, le vol, pour se partager enfin les dépouilles de leurs victimes ». Mais aussi parce que le feuilleton complique singulièrement la donne : la progression de l'intrigue montre que la sauvagerie ne se distribue pas exclusivement dans l'univers pointé par l'incipit, ce que confirme au jour le jour l'évolution du lectorat, où le bourgeois côtoie le barbare. La civilisation et la sauvagerie au coude à coude, là se situait sans doute toute l'immoralité du texte.

Relancée par cet extraordinaire succès public, la vogue indienne se poursuit à Paris à la fin de la Monarchie de Juillet. "Tout le monde s'intéressait aux Sioux, aux Pawnies et aux Delaware ", se souvient Henri Cauvain. ${ }^{(23)}$ En 1845, sur l'initiative du roi Louis-Philippe, s'ouvre au Louvre la Galerie indienne du peintre américain Georges Catlin qui, de 1832 à 1840, a sillonné les Grandes plaines et réalisé près de 500 huiles d'après nature. L'exposition est un triomphe. Des écrivains comme Baudelaire ou George Sand s'enthousiasment pour celui qui a voulu « sauver de l'oubli les traits, les mœurs et les costumes de ces peuplades dites sauvages, et qu'il faudrait plutôt désigner par le nom d'hommes primitifs ".(24) Des philanthropes posent alors la question de la survie des tribus, ${ }^{(25)}$ de plus en plus identifiées, sous l'influence des peintures de Catlin, aux seuls Indiens des plaines. Quelques années plus tard, Louis Hachette publie dans sa Bibliothèque rose les récits et souvenirs du peintre. ${ }^{(26)}$ Quant aux Mohicans, ils poursuivent leur carrière au théâtre, avant que Dumas n'impose une autre acception du terme, celle des figures de la bohème parisienne, 
clôturant ainsi, vers le milieu du siècle, l'influence immédiate du cycle cooperien. ${ }^{(27)}$ Le temps était venu d'autres tribus indiennes.

\section{La Sonora de Louis-Napoléon Bonaparte}

À la différence des Américains, pour qui les guerres apaches constituent l'une des dernières étapes de la conquête de l'Ouest, c'est en remontant du Sud vers le Nord que les Français découvrent ces tribus. ${ }^{(28)}$ La nuance est d'importance. Ascendantes, méridiennes, tendant sans se l'avouer vraiment vers l'ancienne "belle" province, ces représentations expriment d'abord un profond désir de France en Amérique. Tout se passe comme si les Français, exclus du continent par le Nord, tentaient de reprendre pied peu à peu par le Sud. Entre les scintillements de l'or californien, qui attire les migrants au lendemain de 1848, les rêves impériaux d'une France mexicaine et les projets de percement d'un canal dans l'isthme centro-américain, s'ouvre une séquence qui, au mitan du XIXème siècle, marque la dernière tentative d'implantation française sur ce continent. Dès 1848, exilés, migrants et voyageurs sont en effet nombreux à se rendre vers la Californie et le Mexique. Les Français constituent alors la seconde communauté étrangère au Mexique, et nombre de ces voyageurs, comme Gabriel Ferry, Paul Duplessis ou Gustave Aimard, furent autant les pionniers du roman français de l'Ouest américain que les « fourriers » de l'expédition du Mexique. ${ }^{(29)}$ Il faut rappeler ici l'insolite équipée de Gaston Raousset-Boulbon qui, avec une poignée d'aventuriers, tente, en octobre 1852, de prendre pied dans la Sonora. L'indépendance qu'il y proclame devait en fait servir de marchepied à la colonisation française. L'expédition, qui tourne vite au fiasco, s'achève en août 1854 par l'exécution du condottiere par les autorités mexicaines. Mais un «moment mexicain » de l'aventure est perceptible ici, que la littérature, on va le voir, s'efforce presque d'emblée de saisir. ${ }^{(30)}$ L'intervention française au Mexique relance, quelques années plus tard, cet attrait pour l'Amérique centrale : à compter de 1862, on parle en effet beaucoup du Mexique à Paris. Des périodiques comme la Revue des deux mondes, Le Monde illustré ou Le Tour du monde multiplient les articles, insistant notamment, pour mieux justifier l'intervention fran- pagne du Mexique.

La fin de l'hégémonie

européenne en Amérique du

Nord (1862-1867), Paris,

Economica, 1996.

(30) Sur ce point, voir

Sylvain Venayre, "Le

moment mexicain dans

l'histoire française de

l'aventure (1840-1860)",

Histoire et société de

l'Amérique latine, $\mathrm{n}^{\circ} 7$,

1998, p. 123-137, et plus

généralement sa thèse,

L'avènement de l'aventure.

Les figures de l'aventure

lointaine dans la France des

années 1850-1940,

université de Paris-I,

2000, à paraître chez

Aubier en 2002. Voir

aussi Ray Allen

Billington, "The Image

of the Southwest in

Early European

Westerns", in The

American Soutbreest :

Image and Reality,

Berkeley, University of

California Press, 1980. 
(31) Aristarco Regalado Pinedo, El Imaginario Mexicano en Francia, 1861-1867, maîtrise d'histoire, Universidad de Guadalajara/Rennes2, 2001.

(32) F. Nye, $L a$

Sonora : étendue, population, climat, produit du sol, mines, tribus indiennes, traduit de l'anglais et accompagné de notes, suivi d'une notice sanitaire et géographique sur la ville et le port de Panama, par A. De

Zeltner, consul de France à Panama, Paris, Bureau de la presse britannique, 1864.

(33) Ibid., p. 10.

(34) Ibid., p. 45.

(35) Ibid., p. 45.

(36) Ibid., p. 49

(37) Louis-Laurent Simonin, L’bomme américain. Notes sur les Indiens des États-Unis, accompagné de deux cartes, Paris,

Arthus Bertrand, 1870,

p. 16. Les autres récits

de Simonin (surtout

connu pour avoir assisté

à l'entrevue de Fort

Laramie en 1867) ont

paru en 1867 et 1868

dans Le Tour du monde et

La Rerue nationale, puis

dans de très nombreux

volumes. Voir par

exemple Une excursion

chez les Peaux-Rouges,

Paris, Challamel Aîné,

1869, et Le Grand

Ouest des États-Unis.

Les pionniers et les Peaux-

Rouges, Paris,

Charpentier, 1869.

\section{(38) Pour une}

approche d'ensemble concernant la littérature western en France, voir les travaux de Paul

Bleton : "Où est

l'Ouest ? Histoire du roman western en France”, in P. Bleton et R. Saint-Germain, Les hauts et les bas de l'imaginaire western, çaise, sur l'insécurité des marges septentrionales du pays, la cruauté des tribus apaches et l'incapacité des autorités mexicaines à les maitriser. ${ }^{(31)}$ Cet attrait pour la Sonora et la frontière nord s'explique tout autant par les importantes richesses minières de la région (mercure, or, fer, plomb, cuivre) que par le retrait momentané des États-Unis, alors en pleine guerre civile. En 1864, les services diplomatiques français font traduire une étude nord-américaine portant sur cette région, qu'ils demandent au consul de France à Panama de compléter par une notice sanitaire et géographique sur cette ville et ce port Pacifique. ${ }^{(32)}$ On perçoit dans un tel document les rêves de grandeur américaine qui peuvent alors germer chez certains. Ceux d'une nouvelle Amérique française, sorte d'immense empire latin en Amérique centrale, entre Sonora et Panama, Rio Grande au nord et forêt du Darien au sud.

Or l'Apache, que les Français découvrent dans les mêmes années, est rapidement perçu comme un obstacle et une menace à cet ambitieux programme. "Le voisinage des Apaches et leurs fréquentes incursions nuisent puissamment à son développement » et limite sa population, lit-on dans ces documents consacrés à la Sonora. ${ }^{(33)}$ À l'inverse des paisibles Yakis, indiens-paysans, ou des nombreuses tribus qui furent dans le passé des auxiliaires volontaires ou involontaire de la civilisation, l'Apache apparaît d'emblée comme un être inassimilable, un méchant sauvage, sans récupération possible. Le portrait qu'on dresse de lui est aussi radical qu'absolu, étonnant à plus d'un titre pour qui connaît celui des voyous des années 1900. "L'Apache est naturellement violent, orgueilleux, cruel et capricieux. " ${ }^{(34)} C^{\prime}$ est un individu 
errant, porté par des plaisirs ou des passions qu'il ne maîtrise pas, comme la gloutonnerie. "Le plus grand plaisir des Apaches est la danse. " ${ }^{(35)}$ Sa cruauté et son manque de sens moral font frémir : les ennemis sont torturés, les femmes maltraitées, les vieillards abandonnés. "L'Apache vit en définitive à l'état sauvage, ne reconnaissant d'autre loi que la force. " ${ }^{(36)}$ Les conclusions s'imposent d'elles-mêmes : seule l'élimination radicale de ces tribus permettra de pacifier et civiliser ces régions. On retrouve les mêmes appréciations quelques années plus tard, en 1869, dans le rapport rédigé par l'ingénieur marseillais LouisLaurent Simonin pour le ministre de l'Instruction, Victor Duruy. Celuici avait confié à Simonin, bon connaisseur de l'Ouest américain, mais aussi expert-géologue traquant pour le compte du gouvernement français les richesses minérales de la planète, une mission d'ethnologie et de linguistique pour éclairer les origines de "l'homme américain ». Dans l'épais dossier qu'il remet à la bibliothèque impériale, Simonin évoque les irréductibles Apaches parmi les cinq grandes nations du Sud (avec les Kiowas, Arapaos, Comanches et Cheyennes). ${ }^{(37)}$

C'est dans ce même contexte qu'apparaissent les premiers romans français de l'Ouest américain et, dans leur sillage, les premiers Apaches de papier. ${ }^{(38)}$ Dès 1853 , sont en effet publiés, dans une étroite synchronie, les récits de Gabriel Ferry et de Louis-Xavier Eyma, ${ }^{(39)}$ suivis quelques années plus tard de ceux d'Emile-Henri Chevalier, de Paul Duplessis et de Gustave Aimard. ${ }^{(40)}$ Un genre est lancé, auquel le dynamisme de l'édition française sous le Second Empire donne rapidement toute sa mesure. Dès les années 1860, se multiplient titres et collections, à l'image des prolixes "Drames de l'Amérique du Nord", d'abord publiés par Michel Lévy, puis repris par Bourdillat, Poulet-Malassis ou Lécrivain et Toubon, ou encore de la série concurrente, "Les Drames du Nouveau Monde", publiée à compter de 1864 chez l'éditeur P. Brunet. Dans cette véritable explosion du roman indien que constitue le Second Empire, la production nationale ne suffit bientôt plus. Dès la fin de la période, apparaissent ainsi les premières traductions de l'Irlandais Mayne Reid.

Pourtant, plus que l'imaginaire canadien ou celui de la prairie que certains romanciers comme Émile Chevalier s'emploient à prolonger, c'est
Montréal, Triptyque, 1997 ; "Western Fiction in French Popular Literature", Paradoxa, vol. 4, n 9, 1998 ; et son livre à paraître en 2002 : Ouesterne. Ni interne, ni externe... : la place de la Frontier dans l’imaginaire français.

(39) Gabriel Ferry, Le coureur des bois ou les chercheurs d'or, Paris, Cadot, 1853 ; La vie sauvage au Mexique. Costal l'Indien ou les lions mexicains, Paris,

Librairie illustrée, sd. (circa 1855) ; LouisXavier Eyma, Les deux Amériques, histoire, moeurs et voyages, Paris, Giraud, 1853 ; Les Peaux-Rouges, scènes de la vie des Indiens, Paris, Giraud, 1854.

(40) Émile-Henri Chevalier, La fille des Indiens rouges, Paris, Michel Lévy, 1856 ; Paul Duplessis, La Sonora, Paris, Cadot, 1858 ; Gustave Aimard, 
Les trappeurs de l'Arkansas, Paris, Amyot, 1858 ; L'éclaireur, Paris, Amyot, 1859.

(41) Sur Gustave

Aimard, voir le dossier établi par Le Rocambole, $\mathrm{n}^{\circ} 13,2000$, p. 7-116.

Voir aussi la thèse citée de $S$. Venayre et la préface de Matthieu Letourneux aux

Trappeurs de l'Arkansas et autres romans de l'Ouest, Paris, Laffont, 2001.

(42) Bénédict-Henri Revoil, "La Sonora de M. de Raousset-

Boulbon”, L'Illustration, $\mathrm{n}^{\circ} 520,12$ février 1853.

(43) Relation de la captivité de Mme Jane Adeline Wilson, parmi les Indiens camanches [sic], suivie de l'bistoire de la dame Forrester et de sa

famille surprise par les sauvages, Paris, impr. Durand, 1855. Diverses rééditions suivent, dont une avec complainte (Complainte sur l'bistoire de Jane Adeline Wilson, Durand, 1856), une contrefaite à Montpellier en 1860 (impr.

Vve Julien, 1860) et une reprise par les Veillées du foyer, bibliothèque morale et populaire (Tarbes, impr. J.A. Fouga, 1862).

bien l'horizon mexicain qui nourrit les récits de ces auteurs, dont beaucoup ont personnellement pris part à l'exploration de ces régions. C'est le cas de Ferry bien sûr, qui sillonne le Mexique et la Californie de 1830 à 1850, mais aussi de Paul Duplessis, qui voyage dans la Sonora, ou encore de Gustave Aimard (Olivier Gloux de son vrai nom), dont rien ne prouve qu'il ait participé comme il l'affirme à l'expédition de Raousset-Boulbon, mais qui séjourna effectivement à plusieurs reprises au Mexique dans les années 1840 , où il se fit coureur des bois. ${ }^{(41)}$ Pour ces hommes et leurs émules, l'équipée héroïque de la Sonora constitue une sorte de grand récit fondateur, dépositaire d'une large part de leur imaginaire. Auteur à succès de nombreux récits de Peaux-Rouges publiés jusqu'à la fin du XIXème siècle, le romancier Bénédict-Henri Revoil ne fut-il pas l'un des premiers historiographes de l'expédition de Raousset-Boulbon ? ${ }^{(42)}$

On ne s'étonnera donc pas que, davantage qu'aux Pieds-Noirs ou aux Sioux, ces récits fassent la part belle à ces Indiens du Sud qui rôdent dans le no man's land désertique séparant le Mexique des ÉtatsUnis. Au reste, c'est vers ces contrées que tend alors à se déplacer la dynamique de la frontière. À Paris, la vogue est aussi aux tribus du désert. En 1855, par exemple, est largement diffusé un canard relatant par le menu plusieurs récits de massacres et d'enlèvements au Texas par d'abominables Indiens comanches, pillards, violeurs et assassins. ${ }^{(43)}$ Les romanciers participent largement de cette découverte, qui profite dans un premier temps surtout aux Comanches. Peu d'Apaches chez Eyma, par exemple, qui s'étend en revanche longuement sur les tribus comanches. 
Mais un partage s'opère vite entre ces deux tribus, qui s'exprime dès les premiers romans de Gabriel Ferry. Ouvrage fondateur en ce qu'il explicite assez clairement le dernier grand rêve américain de la France (auquel s'emploie le héros, le trappeur canadien Bois-Rosé, qui essaie de tromper sa nostalgie dans ces espaces tropicaux), Le coureur des bois met en scène un diptyque productif. Au Comanche, sauvage moral et assimilable, prototype du bon Indien avec lequel une certaine intelligence s'avère possible, s'oppose l'Apache, être cruel et sournois, renégat et maraudeur, obstacle absolu à toute solution raisonnée. On retrouve le même tableau deux ans plus tard dans Costal l'Indien, où Ferry introduit quelques "diables rouges " apaches, hostiles à toute idée de civilisation. ${ }^{(44)}$

Mais c'est surtout avec Gustave Aimard qu'une telle représentation prend toute sa dimension. Plus sûrement que Ferry, dont l'imaginaire circule des jungles tropicales aux déserts du Nord, Aimard ancre son inspiration dans la Sonora, cette "Apacheria " où il a plusieurs fois séjourné. ${ }^{(4)}$ " Deux fois, il a été attaché par les Apaches au poteau de torture ", affirme d'ailleurs l'avant-propos des Trappeurs de l'Arkansas. Faux, cruels, mauvais, les Apaches incarnent pour Aimard le dernier degré de la sauvagerie. Ne sont-ils pas, comme il le note dans L'éclaireur, les parias du monde indien, ceux que l'on n'invite pas au grand conseil des tribus? Sans doute faut-il leur reconnaître quelques qualités, comme la bravoure et la force, mais leurs tares sont innombrables : "Ils sont ivrognes, voleurs et pillards, sans foi ni loi [...] hautains, cauteleux, rusés, trompeurs, le regard chercheur [...] d'une saleté dégoûtante et même honteuse. » ${ }^{(4)}$ Quelques années plus tard, il offre de ceux qui constituent « la nation la plus féroce et la plus barbare de toutes les savanes de l'Ouest » un portrait apocalyptique : "Ces tyrans du désert ne vivent que de meurtres, viols, pillages, tortures et incendies. Ils attaquent les blancs, les rouges et les métis, sans distinction, et sans autre raison que leur implacable haine contre tout ce qui est étranger. Et lorsqu'ils n'ont pas d'autres ennemis à combattre, ils s'égorgent et se massacrent entre eux, pour le seul plaisir de voir couler le sang. " ${ }^{(47)}$

Ces images terribles se diffusent rapidement dans la France du milieu du siècle. Ce sont elles qu'enregistre par exemple Pierre Larousse, dont
(44) Gabriel Ferry, Costal l'Indien, op. cit., p. 64-69.

(45) Gustave Aimard, Valentin Guillois, Amyot, 1862, p. 349.

(46) Gustave Aimard, L'éclaireur (1859), Paris, Laffont, 2001, p. $802-803$.

(47) Gustave Aimard, Les Peaux-Rouges de Paris, Paris, Dentu, 1888, p. 312-313. 
(49) Paul Toutain,

(48) Louis-Xavier

Eyma, Les Peaux-

Rouges..., op. cit., p. 43, 63 et 307.
Un Français en Amérique : yankees, indiens, mormons, Paris, Plon, 1876, p. 104 et $93-98$. le Dictionnaire décrit " la plus belliqueuse de toutes les tribus sauvages du Nouveau-Mexique ", ces bandes de maraudeurs cruels, gloutons, sales et polygames. "Toujours à cheval, toujours en mouvement, ils sont les cosaques du nouveau monde. » Pour Aimard cependant, la monstruosité de l'Apache ne fait sens qu'en regard de la grandeur du Comanche. Car tout n'est pas perdu, chez le romancier, des traditions du bon sauvage, loyal et courageux, qui perdure dans " la race magnifique " et malheureusement condamnée des Comanches. On perçoit dès lors la place centrale qu'occupe l'Apache dans ce système de représentations : figure inqualifiable, archétype du sauvage féroce et sanguinaire, il permet de justifier la stratégie d'élimination menée au nom de la civilisation, tout en témoignant sympathie et regrets à l'égard de ces bons Indiens que l'irresponsabilité des Apaches condamne malgré eux. Plus explicite qu'Aimard, Louis-Xavier Eyma tire rapidement les leçons de cette situation. Il ne fait aucun doute, écrit-il, que «le but forcé de la politique des Américains envers les Indiens est la destruction radicale de ces indigènes ». Dénonçant l'angélisme et les niaiseries sentimentales qui " ont contribué à fausser l'opinion publique sur le compte des Indiens et des sauvages ", il invite à accepter cette solution au nom de la morale et de la civilisation. La cruauté de ces tribus, leurs « instincts féroces » et surtout leur refus de s'assimiler rendent en effet leur destruction nécessaire. ${ }^{(48)}$ "Cette race est décidément bien condamnée à périr ", conclut un voyageur qui rapporte quelques années plus tard certains récits atroces circulant sur les Indiens de la Sonora. ${ }^{(4)}$ 


\section{Les Indiens, la chasse à l'homme et les voyous}

Tout est donc en place à la fin du Second Empire pour l'importation des Apaches en France. Popularisée par ces récits à grande diffusion, la figure de l'Apache comme rebut et obstacle à la civilisation s'accorde en effet assez bien aux nouvelles stratégies d'intégration des classes " inférieures " que l'Empire libéral, puis la jeune République, s'efforcent de promouvoir. Rien de tel, en effet, pour décriminaliser la menace ouvrière, que de la fragmenter en isolant de l'ensemble, désormais moralisé et acquis aux valeurs et aux normes de la société moderne, ces tribus dissidentes et irrécupérables qu'il ne restera bientôt plus qu'à éliminer par le bagne et par la guillotine.

Une dernière série d'événements contribue enfin, dans les années 1880 , à diffuser l'image de ces sauvages hostiles. De 1883 à 1886, se déroulent en effet les dernières guerres indiennes, précisément menées contre les tribus apaches de Geronimo, et l'événement est largement évoqué par les journaux et les périodiques du pays. Trois ans plus tard, en 1889, le Wild West Show de Buffalo Bill s'installe près de la porte Maillot et consacre l'une de ses attractions à la reddition du chef apache. La culture indienne, relève un ethnographe, fait alors à tel point partie du savoir ordinaire des Français "qu'il est difficile d'en parler sans tomber dans des redites et des lieux communs ». ${ }^{(50)}$ Les Apaches sont au cœur de ces représentations. Dans Sapho d'Alphonse Daudet (1888), l'on voit l'un des artistes invités à un bal masqué se déguiser en chef apache. Les textes de Ferry et d'Aimard sont régulièrement réédités ou démarqués dans des collections à grand tirage, ${ }^{(51)}$ et les Apaches abondent encore dans les romans ou les périodiques d'aventures comme Le Journal des voyages. ${ }^{(52)}$

En 1885, l'ethnologue Élie Reclus, frère aîné du célèbre géographe anarchiste, fixe pour la science les traits de la nation apache. ${ }^{(53)}$ " C'est une belle bête féroce que l'Apache ", écrit l'auteur en ouverture ${ }^{(54)}$ d'un portrait-charge qu'il convient de citer un peu plus longuement tant il préfigure par sa construction, ses motifs ou ses conclusions, le discours sans nuance qu'on tiendra quelques années plus tard sur les jeunes délinquants parisiens. Un physique difficile, d'abord, et aisément identifiable : "Masque impassible, traits ridés et flétris ; figure large, nez

(50) J. Fortescue,

Les Indiens Cris de

l'Amérique du Nord,

Société d'ethnographie

de Paris, 1884, p. 31.

(51) Gabriel de

Bellemarre, Les dernières

aventures de Bois-Rosé,

Paris, Hachette, 1899.

(52) Marie Palewska,

"La partie récréative du

Journal des voyages",

Le Rocambole, $\mathrm{n}^{\circ}$ 6, 1999 , p. 30-33.

(53) Élie Reclus, Les primitifs. Études d'ethnologie comparée : byperboréens orientaux et occidentaux, Apaches, monticoles des Nilgherris, Naïrs, Khonds, Paris, C. Chamerot, 1885 , p. $144-167$.

(54) Ibid., p. 145. 
(55) Ibid., p. 146.

(56) Ibid., p. 151.

(57) Ibid., p. 158.

(59) Paul Féval,

Les amours de Paris, Paris,

Comptoir des impri-

meurs unis, 1845 ;

Les couteaux d'or, Paris,

(58) Ibid., p. $168 . \quad$ A. Cadot, 1857. Cette

dernière référence

n'avait pas échappé à la

vigilance de Régis

Messac, qui l'évoque

dans Le détective Novel

et l'influence de la pensée

scientifique, Paris,

Champion, 1929. aplati, pommettes saillantes, bouche trop fendue, lèvres minces $[\ldots]$ yeux légèrement obliques et dont l'éclat vitré rappelle ceux du coyote. " ${ }^{(5)} \mathrm{Un}$ état d'indicible sauvagerie, ensuite, que révèlent la saleté de ce peuple ou ses pratiques alimentaires : "Se jetant sur leurs proies, ils les dévorent encore vivantes : les uns coupent et taillent, les autres arrachent les membres et les déchiquettent, à force de bras, sans plus de souci des souffrances de la victime que le civilisé qui gobe une huître arrosée d'un filet de citron. Aussi les Apaches sont accusés d'anthropophagie. Le fait n'est pas prouvé. ${ }^{(56)}$ Leur état social, très primitif, est dicté par la seule force brute. Dénués de toute moralité, ils maîtrisent à peine le langage et s'expriment plutôt par gestes. Ils " ne vivent guère que de rapines, leurs maraudages se compliquent de rapts et de meurtres ; leurs combats sont moins des luttes que des assassinats. Rapines, meurtres et massacres, ils en tirent gloire ». ${ }^{(57)}$ Lâches, sournois, cruels, ils n'attaquent que sûrs de tuer et «se délectent à faire subir aux prisonniers d'abominables supplices ». "Race errante, affamée, altérée, race traquée et poursuivie, race endurante, rusée et passionnée, indomptable à la fatigue et à la souffrance, l'Apache, peuple loup, aura le sort du loup ", conclut le savant. ${ }^{(58)}$ Faut-il s'étonner, dans ces conditions, que ce texte ait été réédité en 1903 ?

Tandis que se consolident ainsi les représentations de l'Apache en rebut de la civilisation, voire de l'espèce humaine, on voit se multiplier les récits liant explicitement les Indiens et les voyous, ou jouant de la correspondance entre l'Amérique des tribus et le Paris des bas-fonds. Dès 1845, dans Les 
amours de Paris, Paul Féval introduit dans une sordide affaire de succession le sachem Oguah, chef d'une tribu de Cherokees, qui se révèle au bout du compte être le marquis Jean de Maillepré, l'un des héritiers d'une vieille maison seigneuriale. Huit ans plus tard, le même Féval est le premier à forcer vraiment l'usage métaphorique, en important Towah, authentique Pawnie pour sa part, dans les quartiers mal famés de la capitale. Pour accomplir sa vengeance et punir les assassins du " Mayor ", l'Indien mène la traque dans les rues de Paris, jusqu'à ce qu'il retrouve et scalpe les bandits qu'il pourchasse depuis la Californie. ${ }^{(59)}$ Mais c'est plus comme un coureur de pistes, dans la meilleure tradition de Cooper, que comme une figure des bas-fonds que Towah se présente. Vers le milieu du siècle, pourtant, la correspondance devient générale entre l'Indien et le malfrat. En 1860, Alfred Delvau conduit un de ses amis étrangers " chez les Peaux-Rouges ", du côté de la place Maubert, chez " ces sauvages de la civilisation, ces Peaux-Rouges du Paris moderne, qui sont comme les scories de la grande capitale en ébullition de progrès ". "J'ai écrit le mot, rajoute-t-il en se croyant original, et je ne le bifferai pas. Ces gens-là sont les Peaux-Rouges de Paris. " ${ }^{(60)}$ L'usage du terme progresse également pour désigner tout individu menaçant ou hors norme. En 1871, note un lexicologue, il est fréquent de traiter les Communards de "Peaux-rouges ». ${ }^{(61)}$ Dix ans plus tard, c'est comme des "sauvageons indomptables » que les jeunes délinquants sont présentés par un médecin parisien. ${ }^{(62)}$ En 1884 encore, un homme accusé d'avoir tué son beaufrère est dépeint par un magistrat comme un "véritable Apache ", car il était " tatoué des pieds à la tête comme un véritable sauvage ". ${ }^{(63)} \mathrm{Si}$ elle n'a pas encore contaminé le registre littéraire ou médiatique, la métaphore, on le voit, est largement à l'œuvre dans le discours social.

C'est à Gustave Aimard, ou à l'un de ses continuateurs (Les PeauxRouges de Paris paraît en 1888, cinq ans après la mort de l'auteur), qu'il revient donc d'assurer explicitement la jonction littéraire entre la Sonora et la pègre parisienne. ${ }^{(64)}$ Après une première partie très classique, où les héros affrontent diverses bandes d'Apaches dans la région de la Gila, Aimard transpose l'action en plein Paris, à un moment où l'haussmannisation bouleverse l'organisation de la ville. Plagiant le Féval des

(60) Alfred Delvau, Les dessous de Paris, Paris, Poulet-Malassis, 1860, p. 113.

(61) Jean Dubois, Le vocabulaire politique et social en France de 1869 à 1872 à travers les auvres des écrivains, les revues, les journaux, Paris, Larousse, 1962, p. 93-96.

(62) Le National, 13 septembre 1881, cité par Sophie Diehl, La question sécuritaire à Paris, 1880-1885, maîtrise d'histoire, université de Paris-7, 1999, p. 46.

(63) La Gazette des tribunaux, 28 juillet 1884, citée par Frédéric Chauvaud, Les experts du crime. La médecine légale en France au XIXème siècle, Paris, Aubier, 2000, p. 84.

(64) Gustave Aimard, Les Peaux-Rouges de Paris, op. cit. 
(65) Ibid., p. 369.

(66) Jean-Claude

Vareille, Filatures.

Itinéraire à travers les cycles

de Lupin et Rouletabille,

Presses universitaires de

Grenoble, 1980.
Couteaux d'or, il introduit le guerrier comanche Tahera dans une capitale en pleine effervescence. Mais la convergence est ici poussée à son paroxysme. Projeté dans les cloaques et les tapis-francs de la rue des Vertus ou de la place Maubert, l'Indien est bientôt confronté aux escarpes parisiens que dirige le Loupeur, chef suprême de "l'armée roulante ". Par un classique processus d'inversion, ou de monde à l'envers, ce sont bientôt les rôdeurs et les repris de justice qui apparaissent comme les véritables Peaux-Rouges de Paris, ceux que le guerrier Tahera s'emploie à réduire. Le parallèle s'impose donc entre les pillards Apaches de la première partie et les affidés du Loupeur, la Gouape, Caboulot, Fil-en-quatre ou la Marlouze. Aux innombrables vocables indiens dont Aimard essaime tout le début du récit, succèdent les expressions des bas-fonds, portées par les mêmes italiques. Entre les voyous et les Apaches s'établissent d'elles-mêmes d'évidentes correspondances : même immoralité, même sauvagerie, même exotisme de mœurs et de langage : "Bigre ! s’écria le Loupeur, voilà un peuple au milieu duquel je ne voudrais pas vivre, par exemple ! " ${ }^{(65)}$ Il ignorait évidemment qu'il était déjà l'un des leurs.

À ces corrélations de plus en plus transparentes se surajoutent les analogies, classiques elles aussi, entre le monde de la forêt et de la chasse, dont l'Amérique offre une représentation actualisée, et les structures émergentes du roman de détection. L'animalisation des actants, détective en limier, criminel en bête fauve, est en effet un des éléments constitutifs du roman policier. ${ }^{(66)}$ Rarement cérébrale, l'enquête emprunte le plus souvent les voies de 
la poursuite, de la traque, reliant toujours réflexion et action dans la pratique de l'événement. Dans le sillage de Cooper et de Sue, la plupart des feuilletonistes ont ainsi convoqué l'imaginaire de la chasse et le décor de la forêt, transplantés dans celui de la grande ville. «Les forêts vierges de l'Amérique sont moins dangereuses que les forêts vierges de Paris ", avait noté Dumas, ${ }^{(6)}$ et Féval intitule "La Forêt de Paris" un chapitre de ses Habits noirs. La métaphore est bientôt si employée que Théodore de Banville la dénonce dès 1859 comme un insupportable lieu commun littéraire. ${ }^{(68)}$ Elle constitue pourtant cette voie par laquelle les formes plus modernes et finalement très "intellectuelles" du detective novel, dont on pressent dès la décennie 1860 toutes les potentialités, vont peu à peu se cheviller au roman criminel, jusqu'à le transformer par un phénomène de dérivation progressive. Qu'un sachem de l'Ontario se trouve mêlé à un crime au pont de Chatou relève, dans ces conditions, de l'ordre du vraisemblable. ${ }^{(6)}$ Aux Indiens, omniprésents dans cette perspective, se surajoutent bientôt les trappeurs, autres figures de chasseurs ou de pisteurs. ${ }^{(70)}$

Mais les auteurs comme Féval ou Aimard, qui poussent à bout la métaphore, sont aussi contraints d'en signaler les limites. Dans Les couteaux d'or, l'Indien Towah, transplanté dans les quartiers nord de Paris, peine à s'y retrouver. "Il faut le désert pour la chasse à l'homme. Dès la première nuit de son séjour à Paris, Towah, qui avait pris jadis avec beaucoup de soin la mesure du pied de son ennemi, commença à chercher des pistes dans la neige battue le long des rues et des boulevards ; mauvais métier ; abondance de biens nuit : il y a trop de pistes, Towah rentra découragé. " ${ }^{(7)}$ Et s'il finit par retrouver les bandits, ce n'est pas par les méthodes traditionnelles des coureurs des bois. Une trentaine d'années plus tard, Les Peaux-Rouges de Paris abordent de front cette question, dans un face-à-face exemplaire. D'un côté, le Comanche Tahera et ses amis pisteurs, grands "découvreurs de traces » et admirateurs des rastreadores, bien décidés à utiliser les méthodes de la savane : "La forêt parisienne est peut-être plus dangereuse que celle de l'Arizona, mais nous saurons bien, quand il le faudra, y retrouver les traces de nos ennemis. " ${ }^{(72)}$ De l'autre, le policier Pascal Bonhomme, fin limier de la brigade de Sûreté, qui doute des méthodes des chasseurs ("Paris ne res-
(67) Alexandre Dumas, Les Mohicans de Paris, op. cit., p. 2220.

(68) Théodore de Banville, Petites études. Mes Souvenirs, Paris, Charpentier, 1882.

(69) Charles Joliet, Le crime du pont de Chatou, Paris, CalmannLévy, 1886.

(70) Pierre Léonce Imbert, Les trappeurs parisiens au XIXème siècle, Paris, Sagnier, 1878.

(71) Paul Féval, op. cit., p. 126.

(72) Gustave Aimard, Les Peaux-Rouges de Paris, op. cit., p. 343. 
(73) Ibid., p. 244.

(74) Ibid., p. 288.

(75) Ibid., p. 253. semble nullement aux déserts dont vous parlez; les méthodes dont vous vous serviez seraient d'une exécution impossible en France $\left.{ }^{(73)}\right)$, rejoint dans ses convictions par les escarpes parisiens, qui se gaussent des Américains et ne craignent que la police et ses réseaux d'indicateurs. "Je suis fermement convaincu qu'un coureur des bois, si habile qu'il fût, serait très embarrassé s'il lui fallait mettre en pratique ses étranges talents dans les rues de Paris, où toute piste, à la mode des Peaux-Rouges, est impossible ", expose le Loupart, chef des bandits parisiens. ${ }^{(74)}$ Extraordinaire confrontation, où le policier et le malfrat parisiens, dont les appréciations (réminiscences du style Vidocq ?) convergent momentanément sur ce point, récusent le modèle indien et tout ce qui procède en lui du principe indiciaire et du raisonnement inductif. Durant tout un chapitre, " le lecteur assiste aux premiers tâtonnements de la piste de guerre dans la forêt parisienne et aux ébahissements du célèbre policier, complètement dérouté par l'emploi de procédés à lui inconnus ». ${ }^{(75)}$ Autant que deux méthodes, ce sont aussi deux récits qui s'affrontent. Et si l'enquêteur moderne démontre sa supériorité pour déchiffrer un cryptogramme, il doit aussi s'incliner face au très rigoureux relevé des traces des chasseurs, qui conduit au repaire des bandits. À sa manière un peu naïve, c'est tout un pan de l'imaginaire policier que met en scène Aimard dans cette confrontation, et le dosage subtil qui en résulte entre police sociale, police technique et chasse à l'homme. Les Indiens d'Amérique y ont leur part de responsabilité. 
Tout est donc en place vers 1900 pour que l'Apache devienne le nouveau roi de Paris. La période correspond de surcroît à un regain de représentations indiennes, qui transitent à compter de 1907 par la très active édition en fascicule des maisons Eichler et consorts (Sitting Bull, Rouges et Blancs, Buffalo Bill, Texas Jack, Les chefs indiens célèbres, Jim Kannah, Les mystères $d u$ Far West, etc. $\left.{ }^{(76)}\right)$. On aurait tort cependant de ne voir en l'Apache qu'un Indien parmi d'autres, une simple figure exotique qu'on mobilise alors parce que l'Amérique est à la mode. Forts d'une solide culture indienne, les Français de la Belle Époque savaient fort bien ce qui différencie un Cheyenne d'un Delaware et un Sioux d'un Arapaoe. Le choix de l'Apache répondait donc à des motivations précises. Pour les jeunes délinquants, il traduisait assez bien les logiques de rupture qui commandaient leur dissidence. Au contraire des autres prolétaires, Peaux-Rouges pacifiés et désormais parqués dans des réserves en marge $\mathrm{du}$ front de colonisation sociale, eux refusaient de rendre les armes. Exclus des avancées d'une civilisation de plus en plus normative, récusant le travail et les mirages de l'ère industrielle, soucieux avant tout de jouir d'un plaisir qu'on leur mesurait, ils aspiraient à une liberté altière et sans entrave. Guerriers farouches et insoumis, ils lançaient des raids vengeurs contre les défenseurs du procès de civilisation, mais savaient, dans leur lucidité désespérée, leur combat sans lendemain. Quant à leurs adversaires, ils avaient eux aussi des raisons de les traiter d'Apaches. La guérilla incessante menée par ces bandes rebelles venait rappeler à juste titre la fragilité du progrès, le caractère pionnier mais incertain des avancées sociales. Mais le terme valait surtout pour le destin qu'il promettait. Rebut du monde indien, figure honnie et inassimilable, l'Apache ne pouvait espérer le sort des autres sauvages, ces prolétaires assagis que la République peu à peu avait incorporés à son jeu. Contre cette tribu résiduelle, on ne pouvait mener qu'une stratégie d'élimination radicale, exécutant ses chefs à chaque belle prise et reléguant les autres dans les réserves lointaines.
(76) Voir Philippe Mellot, Les maîtres de l'aventure, 1907-1959, Paris, Michèle Trinckvel, 1997. 